\title{
miR-433 reduces cell viability and promotes cell apoptosis by regulating MACC1 in colorectal cancer
}

\author{
JIAXIN LI ${ }^{1}$, XUPING MAO $^{1}$, XING WANG ${ }^{2}$, GANGGANG MIAO $^{1}$ and JIAXIN LI ${ }^{1}$ \\ ${ }^{1}$ Department of General Surgery, Danyang Hospital Affiliated to Nantong University, Zhenjiang, Jiangsu 212300; \\ ${ }^{2}$ Department of Hepatic Surgery, Jiangsu Provincial People's Hospital \\ (The First Affiliated Hospital of Nanjing Medical University), Nanjing, Jiangsu 210029, P.R. China
}

Received June 23, 2015; Accepted September 6, 2016

DOI: $10.3892 / 01.2016 .5445$

\begin{abstract}
MicroRNAs (miRNAs) are reported to have important roles in regulating the progression of numerous human cancers, although little is known regarding the role of miRNAs in colorectal cancer. The present study aimed to investigate the role of microRNA-433 (miR-433) in colorectal cancer. The expression levels of miR-433 and its target gene metastasis associated in colon cancer-1 (MACC1) in colorectal cancer tissues were evaluated using reverse transcription-quantitative polymerase chain reaction and western blotting. Furthermore, flow cytometry and MTT assays were used to examine the apoptosis, cell cycle distribution and viability of human colorectal cancer cells, and luciferase reporter and western blot assays were performed to verify the regulatory mechanism of miR-433 on MACC1. In addition, caspase- 3 and caspase-9 expression were examined using western blotting. It was demonstrated that miR-433 expression was downregulated in colorectal cancer tissues and cell lines. Artificial upregulation of miR-433 in colorectal cancer cell lines using miR-433 mimics revealed that upregulation of miR-433 was able to reduce the viability and promote the apoptosis of colorectal cancer cells by downregulating MACC1. Taken together, these results suggested that miR-433 may have an important role in the pathogenesis of colorectal cancer.
\end{abstract}

\section{Introduction}

Colorectal cancer, which is currently one of the most common malignant diseases, is associated with a high annual incidence

Correspondence to: Dr Jiaxin Li, Department of General Surgery, Danyang Hospital Affiliated to Nantong University, 2 Xinming West Road, Zhenjiang, Jiangsu 212300, P.R. China

E-mail: lijiaxin002@126.com.cn

Abbreviations: miRNA, microRNA; 3'-UTR, 3'-untranslated region; RT-qPCR, reverse transcription-quantitative polymerase chain reaction; $\mathrm{MACC}$, metastasis associated in colon cancer-1

Key words: microRNA-433, metastasis associated in colon cancer-1, proliferation, apoptosis, cell cycle, colorectal cancer of $\sim 1$ million cases (1). Colorectal cancer is undoubtedly a major health threat to the world's population. Despite advances in the screening and treatment of colorectal cancer, which have improved the life expectancy of patients, the prognosis of patients with colorectal cancer remains poor (1). Therefore, understanding the biological mechanisms underlying colorectal cancer progression is important.

Increasingly, studies have shown that aberrant microRNA (miRNA) expression participates in the development of colorectal cancer $(2,3)$. miRNAs are a family of small non-coding RNAs that are able to post-transcriptionally regulate genes involved in various biological processes (4-6). microRNA-433 (miR-433) has been reported to be dysregulated in several malignancies, including ovarian cancer (7), liver cancer (8) and hemopathy (9). Guo et al (10) reported that miR-433 was downregulated in gastric cancer and functioned as a tumor suppressor. However, the roles of miR-433 in colorectal cancer have yet to be elucidated.

Metastasis associated in colon cancer-1 (MACC1) is an oncogene, and its overexpression has been associated with the development and progression of numerous tumors, including gastric carcinoma (11), hepatocellular carcinoma (12), lung adenocarcinoma (13), esophageal cancer (14), glioma (15) and breast cancer (16). Zhen et al (17) demonstrated that MACC1 overexpression resulted in the upregulation of Met and $\beta$-catenin, as well as its downstream genes, including c-Myc, cyclin D1 and matrix metallopeptidase 9, and the upstream gene phospho-glycogen synthase kinase $3 \beta$ (Ser9). In addition, $\mathrm{MACC} 1$ increased the expression of vimentin and suppressed the expression of E-cadherin in colorectal cancer (17).

In the present study, the expression of miR-433 and MACC1 in colorectal cancer was evaluated in order to investigate the role of miR-433 in the development and progression of colorectal cancer. It was demonstrated that miR-433 was able to reduce the viability and induce the apoptosis of colorectal cancer cells by targeting MACC1.

\section{Materials and methods}

Human tissue specimens. A total of 79 patients with colorectal cancer who had undergone routine surgery at Danyang Hospital Affiliated to Nantong University (Zhenjiang, China) between July 2008 and April 2014 were enrolled in the present study. 
Colorectal cancer and the corresponding adjacent tissues were collected from the 79 patients during the routine surgery. All tissues were immediately frozen in liquid nitrogen and stored at $-80^{\circ} \mathrm{C}$. The tumors were classified according to the World Health Organization classification system (18). This study was approved by the Ethical Committee of The Affiliated Hospital of Nantong University, and informed consent was obtained from all patients.

Cell culture. Five human colorectal cancer cell lines (SW480, SW620, HT29, HCT116 and LoVo) were purchased from the American Type Culture Collection (Manassas, VA, USA). The NCM460 normal human colon mucosal epithelial cell line was purchased from INCELL Corporation LLC (San Antonio, TX, USA). All cells were cultured in RPMI-1640 medium or Dulbecco's modified Eagle's medium supplemented with $10 \%$ fetal bovine serum (all Gibco; Thermo Fisher Scientific, Inc., Waltham, MA, USA), $100 \mathrm{IU} / \mathrm{ml}$ penicillin and $100 \mu \mathrm{g} / \mathrm{ml}$ streptomycin at $37^{\circ} \mathrm{C}$ in a humidified incubator containing $5 \%$ $\mathrm{CO}_{2}$.

Isolation of total RNA and reverse transcription-quantitative polymerase chain reaction $(R T-q P C R)$. Total RNA was extracted from tissues and cell lines using TRIzol ${ }^{\circledR}$ reagent (Invitrogen; Thermo Fisher Scientific, Inc.), according to the manufacturer's protocol. Total RNA was quantified using a NanoDrop spectrophotometer (Thermo Fisher Scientific, Inc., Wilmington, DE, USA), after which miRNA and mRNA were reverse transcribed into cDNA using the PrimeScript RT Master Mix (Perfect Real Time) (Takara Biotechnology Co., Ltd., Dalian, China). The expression levels of miRNAs were assessed using TaqMan miRNA assays with TaqMan ${ }^{\circledR}$ Universal Master Mix II (Thermo Fisher Scientific, Inc., Waltham, MA, USA), according to standard protocol. U6 small nuclear RNA was used for normalization. The primer sequences for miR-433 were as follows: RT primer, GTC GTATCCAGTGCAGGGTCCGAGGTGCACTGGATACGA CGAATAATG; forward primer, 5'-TGCGGTACGGTGAGC CTGTC-3'; and reverse primer, 5'-CCAGTGCAGGGTCCG AGGT-3'. One-tenth of the RT reaction was used for qPCR using the TaqMan 2X Universal PCR Master Mix (Thermo Fisher Scientific, Inc., Waltham, MA, USA) on the ABI 7500 Fast Real-Time PCR system (Applied Biosystems; Thermo Fisher Scientific, Inc., Waltham, MA, USA) and with the following cycling conditions: $95^{\circ} \mathrm{C}$ for $10 \mathrm{~min}$, followed by 40 cycles of $95^{\circ} \mathrm{C}$ for $15 \mathrm{sec}$ and $60^{\circ} \mathrm{C}$ for $60 \mathrm{sec}$. Primer sequences for MACC1 were as follows: Forward, 5'-TTCTTT TGATTCCTCCGGTGA-3' and reverse, 5'-ACTCTGATG GGCATGTGCTG-3'. Primer sequences for GAPDH were as follows: Forward, 5'-GGTGAAGGTCGGAGTCAACG-3' and reverse, 5'-CAAAGTTGTCATGGATGHACC-3'. The relative mRNA expression levels of MACC1 were normalized to GAPDH using the $2^{-\Delta \Delta \mathrm{Cq}}$ method (19).

Transient transfection. Colorectal carcinoma and NCM460 cells were seeded into 6 -well plates at a density of $2 \times 10^{5}$ cells $/ \mathrm{ml}$ per well. Oligonucleotide hsa-miR-433 mimics (miR-433) and normal control (NC; miR-control) oligonucleotides were purchased from Shanghai GenePharma, Co., Ltd. (Shanghai, China). The cells were transfected with hsa-miR-433 or
miR-control at a final concentration of $100 \mathrm{nM}$ using Lipofectamine 2000 (Invitrogen; Thermo Fisher Scientific, Inc., Waltham, MA, USA).

Cell viability assay. Cells were seeded into 96-well plates (6.0x10 ${ }^{3}$ cells/well). MTT assays (Roche Diagnostics $\mathrm{GmbH}$, Penzberg, Germany) were performed to detect cell viability. Cells were incubated with MTT $(5 \mathrm{mg} / \mathrm{ml}$ per well) for $4 \mathrm{~h}$. Dimethyl sulfoxide was used to dissolve the formazan crystals. PBS was used as a control. Absorbance at $490 \mathrm{~nm}$ was measured using the Infinite M200 PRO multimode microplate reader (Tecan Benelux BVBA, Mechelen, Belgium). Three independent experiments were performed in quintuplicate.

Cell cycle distribution and apoptosis analysis. The cells were seeded into 6-well plates at a density of $2 \times 10^{5}$ cells $/ \mathrm{ml}$ per well and transfected with miR-433 mimics or NC for 48 h. Flow cytometry was performed with an Annexin V-fluorescein isothiocyanate/propidium iodide kit (Miltenyi Biotec $\mathrm{GmbH}$, Bergisch Gladbach, Germany) using the BD FACSCalibur ${ }^{\mathrm{TM}}$ flow cytometer (BD Biosciences, Franklin Lakes, NJ, USA). Three independent experiments were performed in quintuplicate.

Western blot analysis. For western blotting, the cells were harvested and total protein was extracted from the cells using radioimmunoprecipitation assay lysis buffer containing phenylmethylsulfonyl fluoride (Roche Diagnostics, Basel, Switzerland). Total protein was quantified using the bicinchoninic acid protein assay (Beyotime Institute of Biotechnology, Haimen, China). Proteins were separated by $10 \%$ SDS-PAGE and blotted onto polyvinylidene fluoride membranes (Merck Millipore, Darmstadt, Germany). After blocking with 5\% non-fat milk in $1 \%$ TBST, the membranes were incubated overnight at $4^{\circ} \mathrm{C}$ with goat anti-human MACC1 (1:1,000; polyclonal; sc-163595; Santa Cruz Biotechnology, Inc., Dallas, TX, USA), goat anti-human caspase-3 (1:1,000; polyclonal; sc-22140; Santa Cruz Biotechnology, Inc.), goat anti-human caspase-9 (1:1,000; polyclonal; sc-8297; Santa Cruz Biotechnology, Inc.) and goat anti-human GAPDH (1:5,000; polyclonal; sc-20357; Santa Cruz Biotechnology, Inc.). Next, the membranes were washed four times with PBS containing 0.1\% Tween 20 (PBST; Sigma-Aldrich; Merck Millipore). The secondary antibody, rabbit anti-goat (1:5,000; polyclonal; sc-2922; Santa Cruz Biotechnology, Inc.), was then added in PBST for $1 \mathrm{~h}$ at $37^{\circ} \mathrm{C}$. The membranes were then washed three times for $15 \mathrm{~min}$ with PBST, and the secondary antibodies were detected using the Pierce ${ }^{\mathrm{TM}}$ ECL Substrate Western Blot Detection system (Thermo Fisher Scientific, Inc., Waltham, MA, USA). Protein bands were visualized using the Molecular Imager ChemiDoc XRS System (Bio-Rad Laboratories, Inc., Hercules, CA, USA). The integrated optical densities of the bands were quantified using ImageJ software version 1.48u (https://imagej.nih.gov/ ij/; National Institutes of Health, Bethesda, MD, USA).

Bioinformatics analysis. The bioinformatics software microRNA.org (http://www.microrna.org/microrna/) and TargetScan (http://www.targetscan.org/) were used to identify candidate target genes of miR-433. 
Table I. Expression levels of miR-433 in colorectal cancer and corresponding adjacent tissues.

\begin{tabular}{|c|c|c|c|c|}
\hline Characteristics & All patients & miR-433 low expression & miR-433 high expression & P-value \\
\hline Number of patients & 79 & 40 & 39 & \\
\hline Age (years) & & & & 0.930 \\
\hline$<60$ & 30 & 15 & 15 & \\
\hline$\geq 60$ & 49 & 25 & 24 & \\
\hline Gender & & & & 0.206 \\
\hline Male & 45 & 20 & 25 & \\
\hline Female & 34 & 20 & 14 & \\
\hline Histological differentiation & & & & 0.288 \\
\hline Well/moderate & 31 & 18 & 13 & \\
\hline Poorly & 48 & 22 & 26 & \\
\hline Tumor size & & & & $0.008^{\mathrm{a}}$ \\
\hline $\mathrm{T} 1, \mathrm{~T} 2$ & 29 & 9 & 20 & \\
\hline $\mathrm{T} 3, \mathrm{~T} 4$ & 50 & 31 & 19 & \\
\hline Tumor stage & & & & $0.002^{\mathrm{a}}$ \\
\hline I or II & 27 & 7 & 20 & \\
\hline III or IV & 52 & 33 & 19 & \\
\hline
\end{tabular}

${ }^{\mathrm{a}} \mathrm{P}<0.05 . \mathrm{miR}-433$, microRNA-433.

A

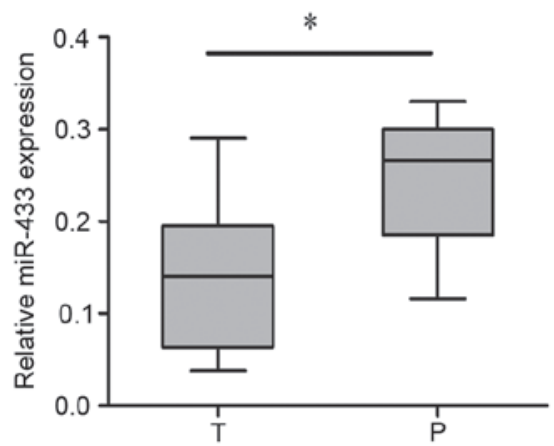

B

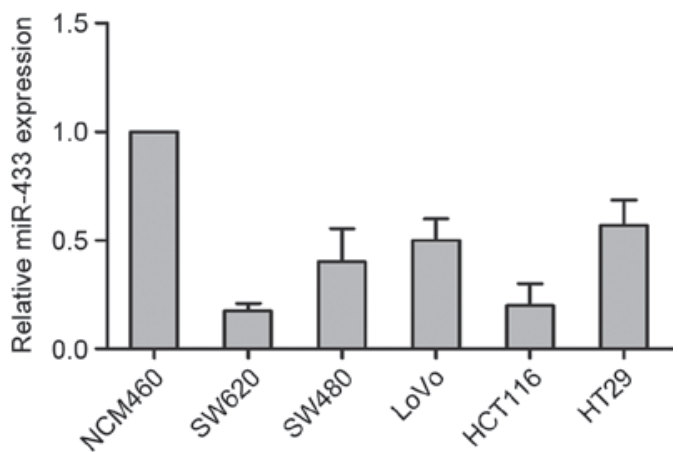

Figure 1. miR-433 is downregulated in human colorectal cancer tissues and cell lines. (A) The expression levels of miR-433 in human colorectal cancer tissues and the corresponding adjacent tissues, relative to U6 small nuclear RNA, were detected by RT-qPCR ( $\mathrm{n}=79$; $\left.{ }^{*} \mathrm{P}<0.0001\right)$. (B) The expression levels of miR-433 in human colorectal cancer cell lines were detected by RT-qPCR. Data are presented as the mean \pm standard error of the mean. T, colorectal cancer tissues; $\mathrm{P}$, corresponding adjacent tissues; miR-433, microRNA-433; RT-qPCR, reverse transcription-quantitative polymerase chain reaction.

Plasmid construction and cell transfection. The 3'-untranslated region (UTR) of MACC1 or a mutant (MUT) sequence with the predicted target sites was inserted into the Kpn I and Sac I sites of the pGL3 promoter vector (GenScript, Nanjing, China). Cells were plated onto 6-well plates at a density of $2 \times 10^{5}$ cells $/ \mathrm{ml}$ per well and transfected with $100 \mathrm{ng}$ pGL3-MACC1 or pGL3-MACC1-MUT and $50 \mathrm{nM}$ miR-433 mimics using Lipofectamine 2000. In addition, the MACC1 gene (synthesized by GenScript) was digested with the Mlu I restriction enzyme and subcloned into the pLV-green fluorescent protein (GFP) plasmid (kindly donated by the Central Laboratory of Nanjing Medical University, Nanjing, China) to form pLV-GFP-MACC1. For amplification of the expression vector, 293T cells (Shanghai Bioleaf Biotech Co., Ltd., Shanghai, China) were transfected with pLV-GFP-MACC1 using the calcium phosphate co-precipitation method.
Colorectal cancer cells were subsequently transfected with pLV-GFP-MACC1 using polybrene $(8 \mu \mathrm{g} / \mathrm{ml}$; Sigma-Aldrich; Merck Millipore).

Dual luciferase assays. Following incubation with pLV-GFP-MACC1 for $48 \mathrm{~h}$, the luciferase activity was analyzed using the Dual Luciferase Reporter Assay system (Promega Corporation, Madison, WI, USA), according to the manufacturer's protocol. The relative luciferase activities were determined by normalizing to the activity of Renilla luciferase.

Statistical analysis. All statistical analyses were performed using the Stata 11 software (StataCorp LP, College Station, TX, USA), and the results were presented with GraphPad Prism software version 4.0 (GraphPad Software, Inc., La Jolla, CA, USA). Continuous variables are expressed as the 
A

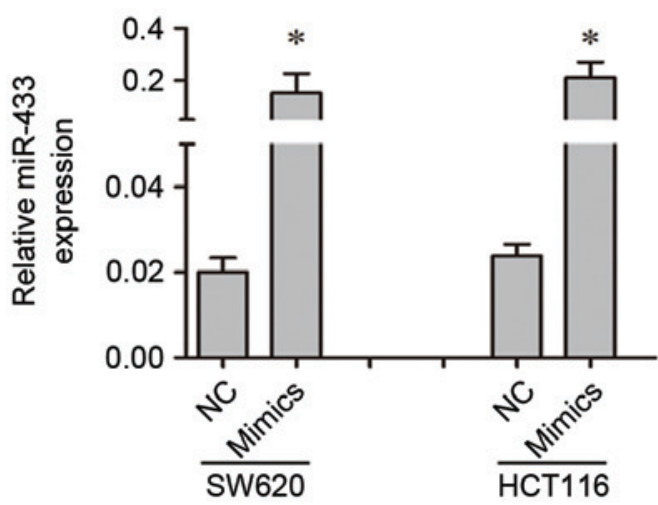

B
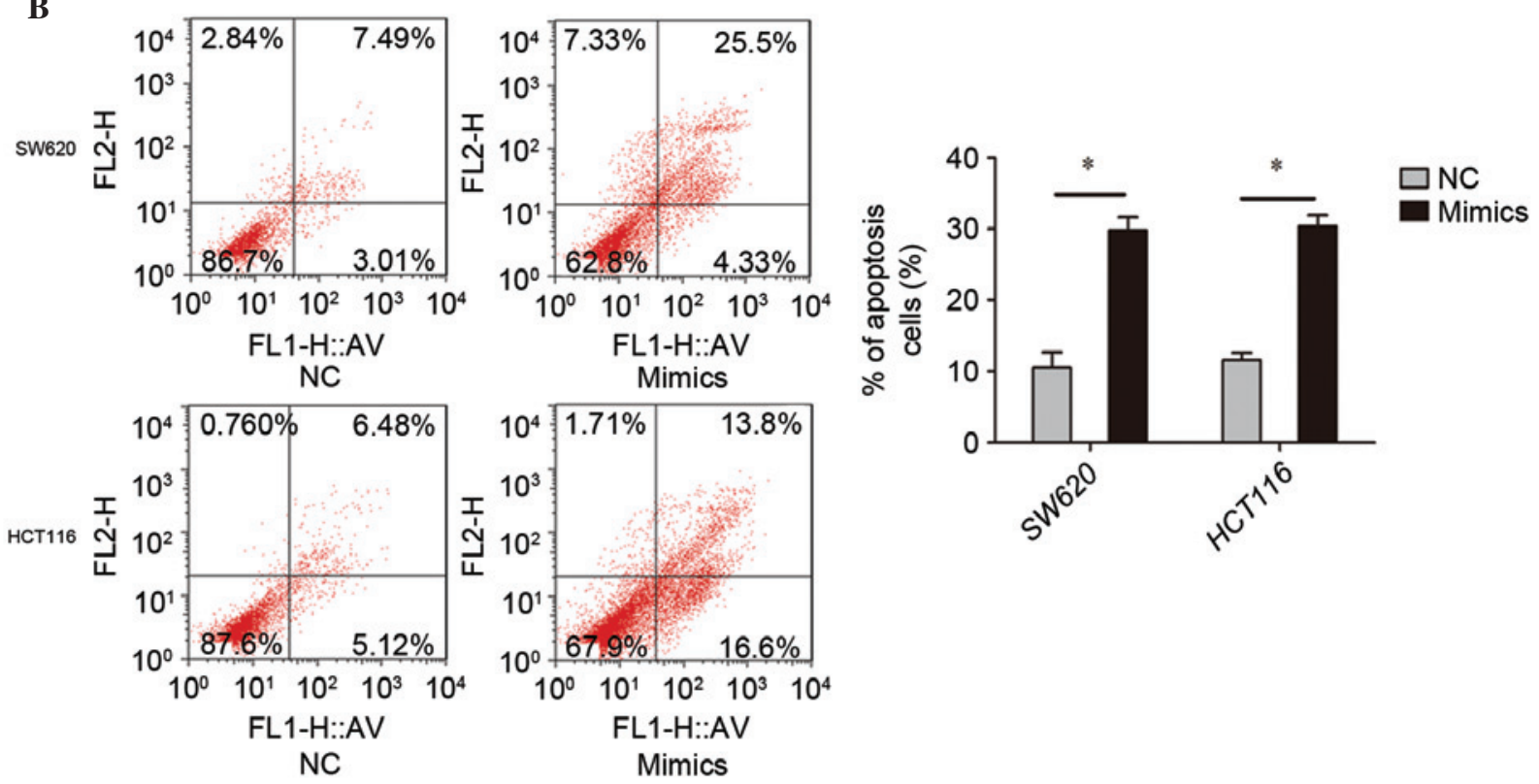

C

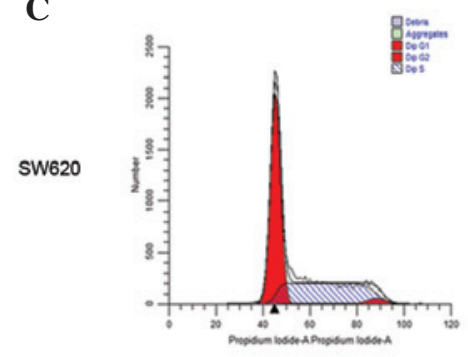

NC

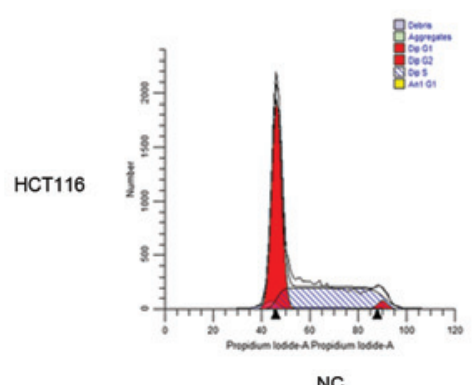

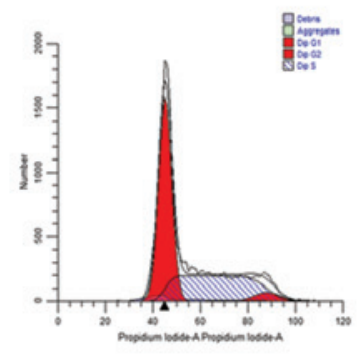

Mimics

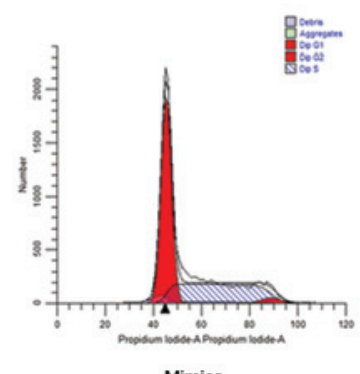

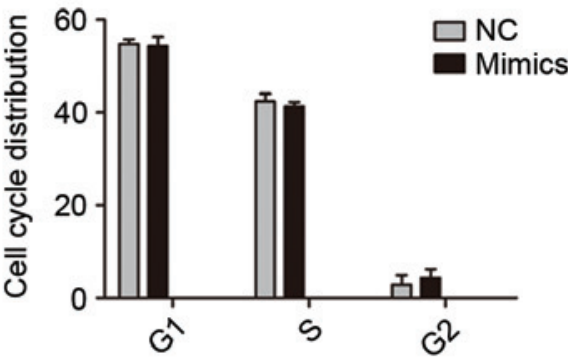

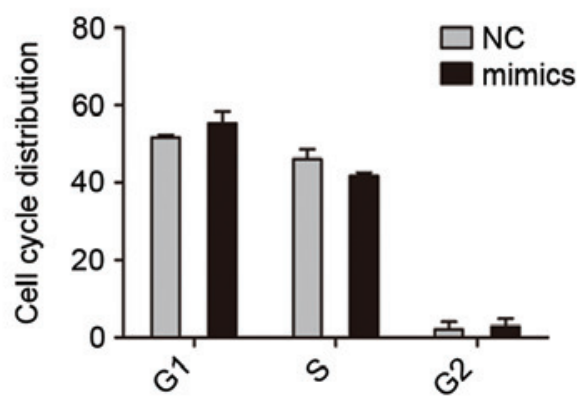

Figure 2. Upregulation of miR-433 promotes cell apoptosis and inhibits cell growth without affecting the cell cycle. (A) The expression level of miR-433 in cell lines transfected with NC or miR-433 mimics was detected by reverse transcription-quantitative polymerase chain reaction. (B) Flow cytometry assays were performed to assess the apoptosis of cells transfected with NC or miR-433 mimics. The apoptosis of SW620 and HCT116 cells was promoted by transfection with miR-433 mimics. The number of apoptotic cells is presented as the mean $\pm \mathrm{SEM}$. * $\mathrm{P}<0.05$ vs. the NC-transfected cells. (C) Flow cytometry was performed to assess the cell cycle distribution of cells transfected with NC or miR-433 mimics. The cell cycle distributions of SW620 and HCT116 cells were not significantly different between the cells transfected with NC and miR-433 mimics. Cell cycle distribution numbers are presented as the mean \pm SEM. Each independent experiment was performed in triplicate. miR-433, microRNA-433; NC, normal control; SEM, standard error of the mean. 
A

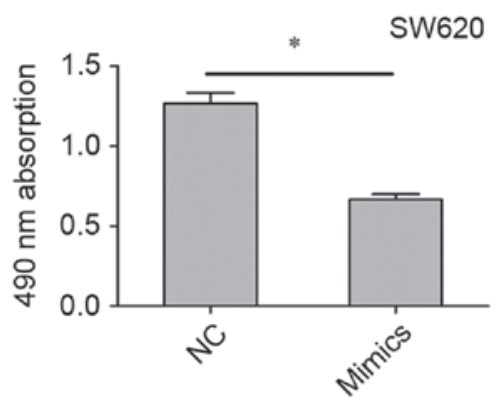

B

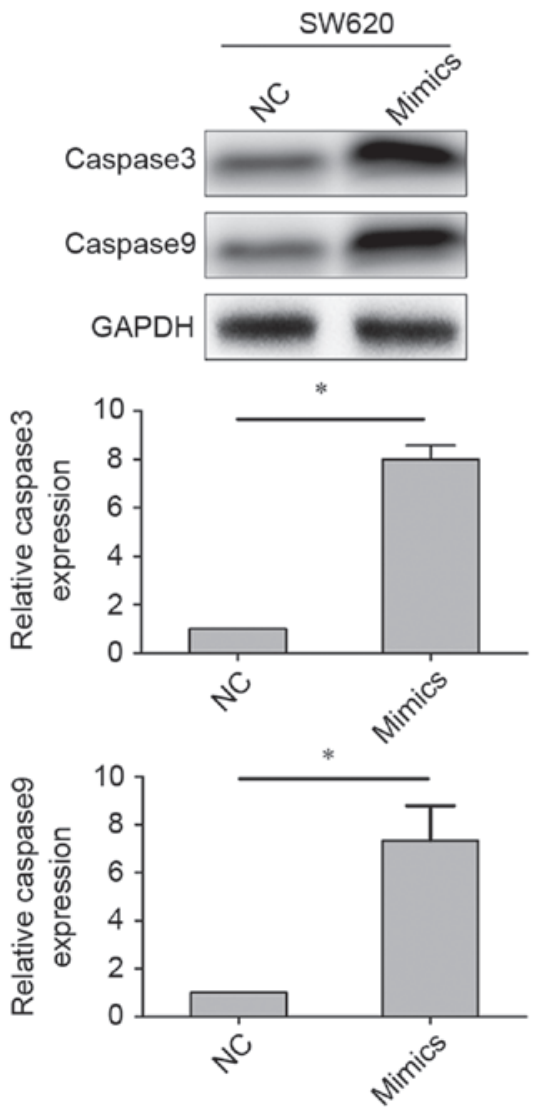

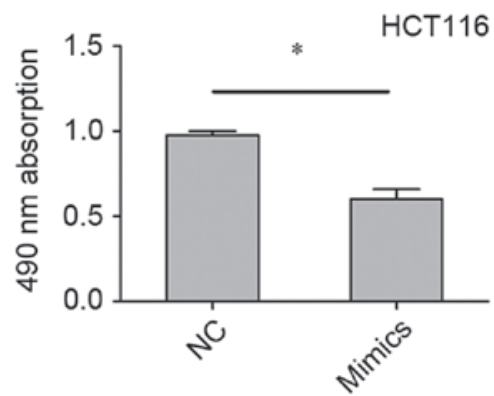
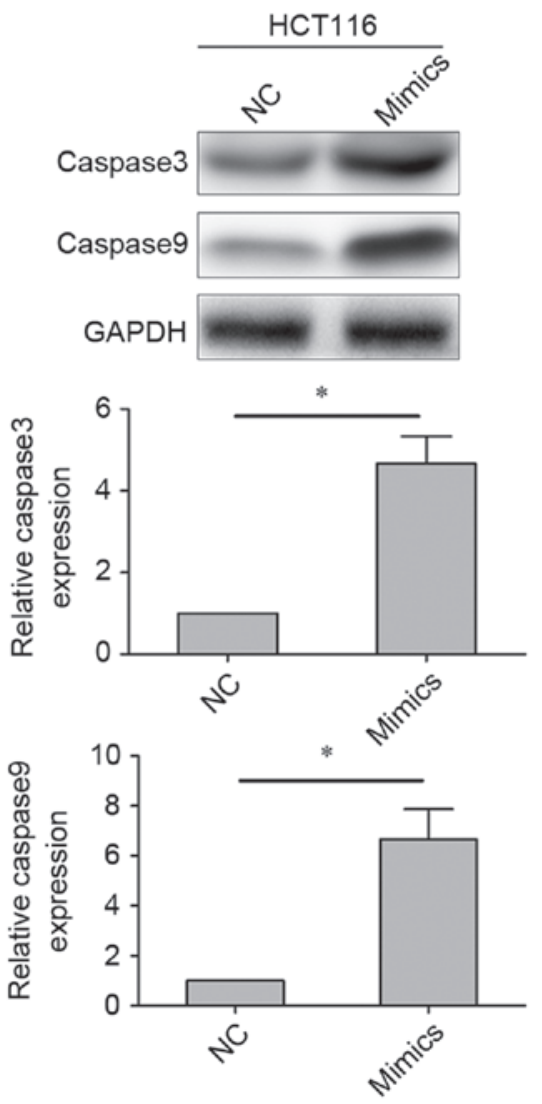

Figure 3. Upregulation of miR-433 reduces the viability of colorectal cancer cells and regulates the expression of caspase-3 and caspase-9. (A) MTT assays were conducted to investigate the viability of SW620 and HCT116 colorectal cancer cells treated with NC or miR-433 mimics. The absorbance at 490 nm is presented as the mean \pm SEM of triplicate experiments. ${ }^{*} \mathrm{P}<0.05$ vs. the NC-transfected cells. (B) The protein expression levels of cleaved caspase- 3 and caspase- 9 were examined by western blotting. ${ }^{*} \mathrm{P}<0.05$ vs. the NC-transfected cells. The relative expression levels are presented as the mean \pm SEM of triplicate experiments. NC, negative control; miR-433, microRNA-433; SEM, standard error of the mean.

mean \pm standard error of the mean. Differences between groups were calculated using Student's t-tests. $\mathrm{P}<0.05$ was considered statistically significant.

\section{Results}

miR-433 is downregulated in human colorectal cancer tissues and cell lines. To explore the role of miR-433 in colorectal cancer, the expression levels of miR-433 in human colorectal cancer samples and their corresponding adjacent tissues were detected by RT-qPCR. It was demonstrated that, as compared with the corresponding adjacent tissues, the expression of miR-433 was significantly decreased in colorectal cancer tissues $(\mathrm{P}=0.00085$; Fig. $1 \mathrm{~A})$. The colorectal cancer tissues were subsequently divided into either the miR-433 low-expression group $(n=40)$ or the miR-433 high-expression group $(n=39)$ (Table I); the median expression level for all samples (median=0.3022; Fig. 1A) was regarded as the cut-off. From analyzing the clinicopathological characteristics of all patients, it was determined that aberrant expression of miR-433 was significantly associated with the tumor size $(\mathrm{P}=0.008$; Table I), suggesting that miR-433 may have an important role the pathogenesis of colorectal cancer. It was then demonstrated that the expression level of miR-433 was markedly reduced in colorectal cancer cell lines compared with the NCM460 normal human colon mucosal epithelial cell line (Fig. 1B), which was consistent with the result of colorectal cancer tissues. These results suggest that miR-433 is downregulated in human colorectal cancer tissues and cell lines.

Upregulation of miR-433 promotes the apoptosis and reduces the viability of colorectal cancer cells without affecting the 
A

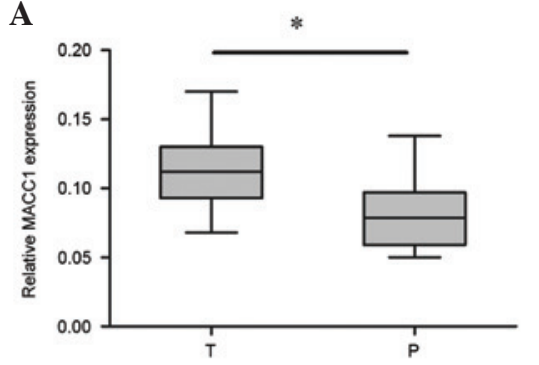

D

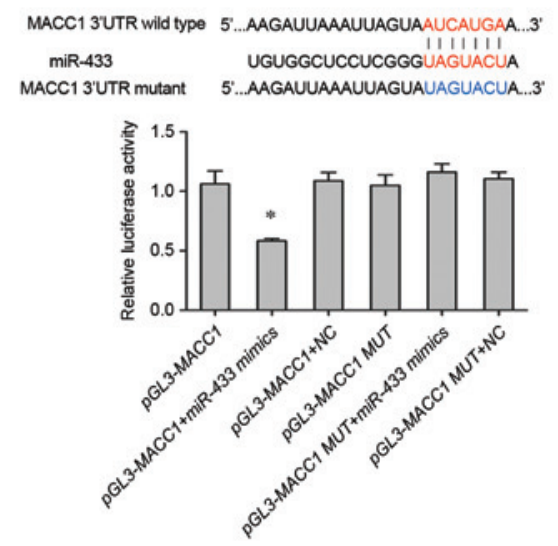

B

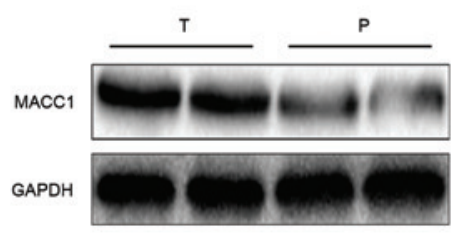

C

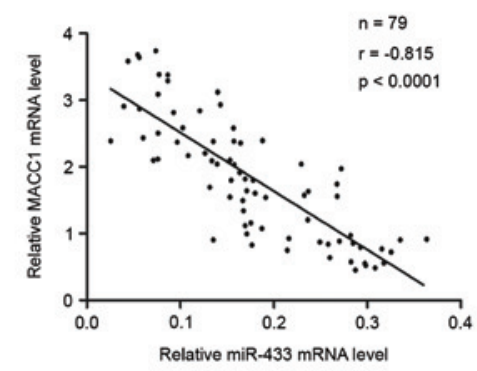

E

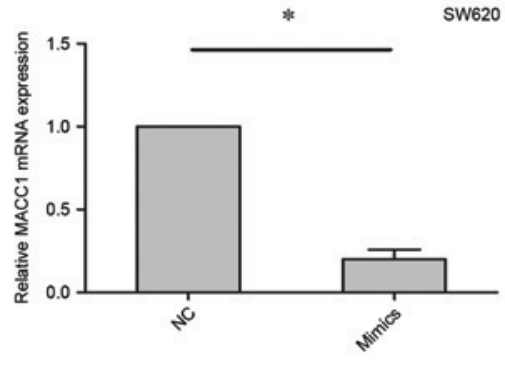

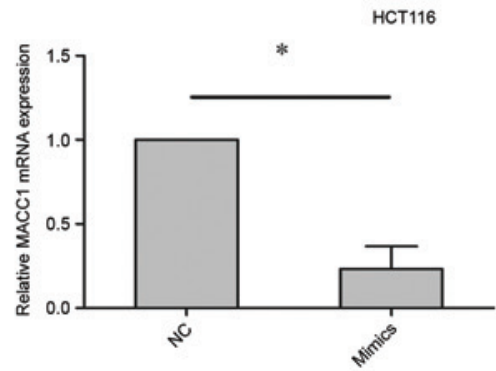

F
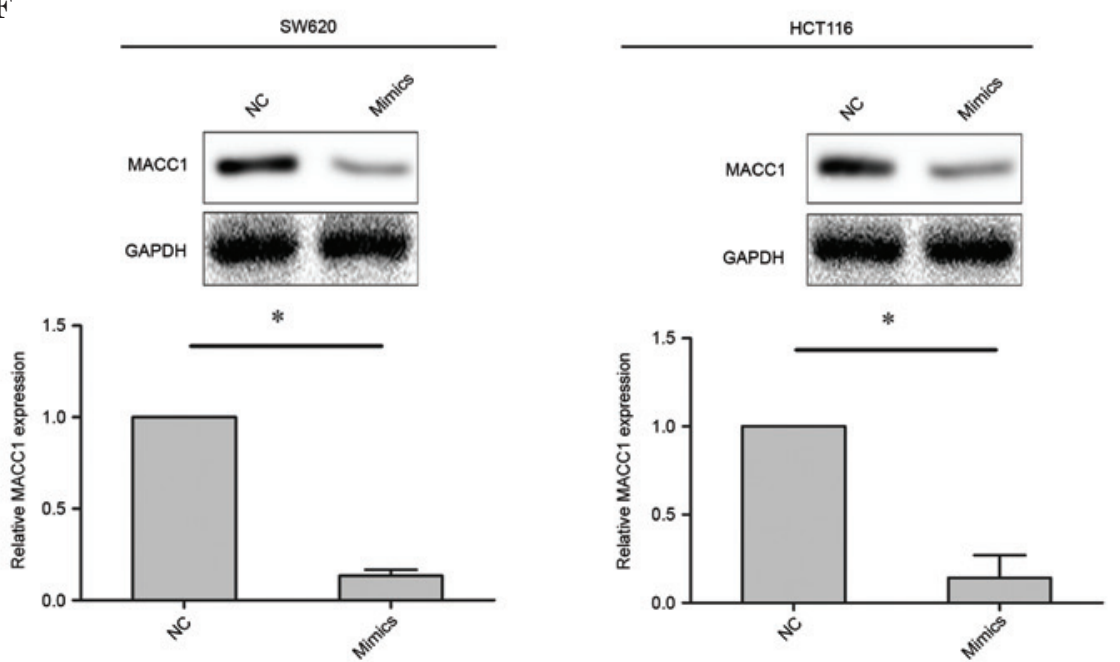

Figure 4. MACC1 is a target gene of miR-433. (A) The mRNA expression levels of MACC1 in human colorectal cancer tissues and corresponding adjacent tissues were detected by RT-qPCR. (B) The protein expression levels of MACC1 were detected by western blotting. (C) A negative correlation was observed between the expression levels of miR-433 and MACC1 mRNA in the tumor samples $(r=-0.815 ; \mathrm{P}<0.0001)$. (D) The potential miR-433 seed region in the $3{ }^{\prime}-\mathrm{UTR}$ of MACC1 mRNA was computationally predicted using TargetScan. SW620 cells were co-transfected with miR-433 mimics or NC and pGL3-MACC1 or pGL3-MACC1-MUT. Luciferase activity was normalized using the ratio of firefly to Renilla luciferase signals. (E) The mRNA expression levels of MACC1 in SW620 and HCT116 colorectal cancer cells transfected with NC or miR-433 mimics were analyzed by RT-qPCR. GAPDH was used as an internal control. (F) The protein expression levels of MACC1 in SW620 and HCT116 cells transfected with NC or miR-433 mimics were analyzed by western blotting. GAPDH was used as an internal control. All experiments were performed in triplicate and the band intensity values were analyzed using ImageJ software. Data are presented as the mean \pm standard error of the mean. ${ }^{*} \mathrm{P}<0.05$. T, colorectal cancer tissues; $\mathrm{P}$, corresponding adjacent tissues; MACC1, metastasis associated in colon cancer-1; NC, normal control; miR-433, microRNA-433; RT-qPCR, reverse transcription-quantitative polymerase chain reaction; MUT, mutant; 3'-UTR, 3'-untranslated region.

cell cycle. To investigate the functional roles of miR-433, cell lines were transfected with miR-433 mimics or NC. RT-qPCR was used to confirm the transfection efficiency (Fig. 2A). Subsequently, the effect of miR-433 on cell apoptosis, the cell cycle distribution and cell viability was assessed. Flow cytometry demonstrated that overexpression of miR- 433 promoted the apoptosis of the colorectal cancer cell lines (Fig. 2B). In addition, it was shown that miR-433 did not affect the cell cycle distribution compared with the control (Fig. 2C). MTT assays were performed to detect the effect of miR-433 on cell viability. The results of MTT assays showed that miR-433 mimics reduced the viability of the colorectal cancer cell lines (Fig. 3A). Furthermore, we investigated the expression of caspase- 3 and caspase- 9 in cells transfected with miR-433 mimics or NC, in order to further assess the effect of miR-433 on cell apoptosis. Western blotting demonstrated that the 
expression levels of caspase- 3 and caspase-9 were increased in cells transfected with miR-433 mimics compared with those transfected with NC (Fig. 3B). These results indicate that upregulation of miR-433 promotes cell apoptosis and reduces cell viability, without affecting the cell cycle of colorectal cancer cells.

$M A C C 1$ is a target gene of $m i R-433$. To investigate the potential role of miR-433 in colorectal cancer cell apoptosis, a bioinformatics analysis, which is commonly used to identify the candidate target genes of miRNAs (20), was performed The bioinformatics analysis focused on candidate genes that have previously been implicated in the regulation of cancer cell apoptosis. microRNA.org and TargetScan were used to select the MACC1 gene, which has previously been associated with colorectal cancer cell proliferation, apoptosis and invasion (17,21). The mRNA and protein expression levels of MACC1 in 79 colorectal cancer samples were investigated using RT-qPCR and western blotting. It was demonstrated that MACC1 was upregulated in the colorectal cancer tissues compared with the corresponding adjacent tissues (Fig. 4A and B). In addition, an inverse correlation was observed between the expression levels of miR-433 and MACC1 ( $\mathrm{r}=-0.815$; $\mathrm{P}<0.0001$; Fig. 4C) in colorectal cancer tissues.

To further evaluate whether the inhibitory effect of MACC1 induced by miR-433 mimics was dependent on the binding of miR-433 to the 3'-UTR of MACC1, the 3'-UTR fragment of MACC1 containing the predicted binding site was cloned into the pGL3 luciferase reporter vector (pGL3-MACC1). The 3'-UTR fragment containing the MUT sequence was also cloned into the pGL3 luciferase reporter vector as a control group (pGL3-MACC1-MUT). Dual luciferase reporter assays showed that the luciferase activity was decreased in cells transfected with miR-433 mimics and pGL3-MACC1 vectors, as compared with the control- and pGL3-MACC1-MUT-transfected cells (Fig. 4D). Furthermore, the mRNA and protein expression levels of MACC1 in cells transfected with miR-433 mimics or control were investigated using RT-qPCR and western blotting. Marked inhibition of MACC1 expression was observed in cells transfected with miR-433 mimics compared with control, suggesting that miR-433 negatively regulates MACC1 in colorectal cancer cell lines (Fig. 4E and F). These findings suggest that the MACC1 gene is a target of miR-433.

\section{Discussion}

Colorectal cancer is one of the most common causes of cancer-associated mortality worldwide (1). miRNAs have been reported to have important regulatory roles in the pathogenesis of colorectal cancer $(2,3)$. miR-378 suppresses the proliferation and induces the apoptosis of colorectal cancer cells by targeting BRAF (22). Regulation of ubiquitin-like PHD and RING finger domain-containing protein 1 by miR-9 modulates colorectal cancer cell proliferation and apoptosis (23). The present study aimed to provide evidence for miR-433 targeting MACC1 in the regulation of the viability and apoptosis of colorectal cancer. It was demonstrated that the expression level of miR-433 was downregulated in colorectal cancer tissues compared with the corresponding adjacent tissues. Furthermore, the upregulation of miR-433 in colorectal cancer cells using miR-433 mimics was shown to reduce the viability and promote the apoptosis of colorectal cancer cells. In addition, an inverse correlation between the expression levels of miR-433 and MACC1 was detected in 79 colorectal cancer tissues.

MACC1 is recurrently upregulated in colorectal cancer, and numerous studies have reported that MACC1 significantly contributes to lymph node metastases, an advanced TNM stage and tumor size (20,21); thus suggesting that MACC1 has an important role in the development of colorectal cancer. MACC1 is a newly identified key regulator of hepatocyte growth factor-Met signaling and has been associated with the progression and prognosis of various carcinomas (15). In a previous study, high MACC1 expression was an independent prognostic indicator for reduced overall survival in patients with colorectal cancer (24). Furthermore, overexpression of MACC1 has been reported to promote the metastasis and recurrence of colorectal cancer (24), and it was associated with peritoneal dissemination and a higher TNM stage (25). The results of the present study suggested that miR-433 regulates the function of MACC1 by binding to its 3'-UTR.

In conclusion, the present study demonstrated that downregulation of miR-433 in colorectal cancer was markedly associated with cancer development. Upregulation of miR-433 in colorectal cancer cell lines promoted cell apoptosis and reduced the cell viability. Furthermore, miR-433 was shown to induce the apoptosis of colorectal cancer cells by regulating the expression of MACC1, which has a crucial role in the development of colorectal cancer. Because of the limited number of colorectal cancer samples and cell types in the present study, further studies investigating the potential role of miR-433 in the development of colorectal cancer are required. In addition, future studies should investigate whether the miR-433-MACC1 pathway might be exploited in a therapeutic approach for the treatment of colorectal cancer.

\section{References}

1. Meyerhardt JA and Mayer RJ: Systemic therapy for colorectal cancer. N Engl J Med 352: 476-487, 2005.

2. Wang YX, Chen YR, Liu SS, Ye YP, Jiao HL, Wang SY, Xiao ZY Wei WT, Qiu JF, Liang L, Liao WT and Ding YQ: MiR-384 inhibits human colorectal cancer metastasis by targeting KRAS and CDC42. Oncotarget, doi: 10.18632/oncotarget.12704, 2016 (Epub ahead of print).

3. Wei W, Yang Y, Cai J, Cui K, Li RX, Wang H, Shang X and Wei D: mir-30a-5p suppresses tumor metastasis of human colorectal cancer by targeting ITGB3. Cell Physiol Biochem 39: 1165-1176, 2016

4. Shao Y, Zhang SQ, Quan F, Zhang PF and Wu SL: MicroRNA-145 inhibits the proliferation, migration and invasion of the human TCA8113 oral cancer line. Oncol Lett 6: 1636-1640, 2013.

5. Yang L, Wang YL, Liu S, Zhang PP, Chen Z, Liu M and Tang H: miR-181b promotes cell proliferation and reduces apoptosis by repressing the expression of adenylyl cyclase 9 (AC9) in cervical cancer cells. FEBS Lett 588: 124-130, 2014.

6. Billeter AT, Barnett RE, Druen D, Polk HC Jr and van Berkel VH: MicroRNA as a new factor in lung and esophageal cancer. Semin Thorac Cardiovasc Surg 24: 155-165, 2012.

7. Weiner-Gorzel K, Dempsey E, Milewska M, McGoldrick A, Toh V, Walsh A, Lindsay S, Gubbins L, Cannon A, Sharpe D, et al: Overexpression of the microRNA miR-433 promotes resistance to paclitaxel through the induction of cellular senescence in ovarian cancer cells. Cancer Med 4: 745-758, 2015.

8. Yang Z, Tsuchiya H, Zhang Y, Hartnett ME and Wang L: MicroRNA-433 inhibits liver cancer cell migration by repressing the protein expression and function of cAMP response element-binding protein. J Biol Chem 288: 28893-28899, 2013. 
9. Lin X, Rice KL, Buzzai M, Hexner E, Costa FF, Kilpivaara O, Mullally A, Soares MB, Ebert BL, Levine R and Licht JD: miR-433 is aberrantly expressed in myeloproliferative neoplasms and suppresses hematopoietic cell growth and differentiation. Leukemia 27: 344-352, 2013

10. Guo LH, Li H, Wang F, Yu J and He JS: The tumor suppressor roles of miR-433 and miR-127 in gastric cancer. Int J Mol Sci 14: 14171-14184, 2013

11. Ge SH, Wu XJ, Wang XH, Xing XF, Zhang LH, Zhu YB, Du H, Dong B, Hu Y and Ji JF: Over-expression of metastasis-associated in colon cancer-1 (MACC1) associates with better prognosis of gastric cancer patients. Chin J Cancer Res 23: 153-159, 2011.

12. Xie C, Wu J, Yun J, Lai J, Yuan Y, Gao Z, Li M, Li J and Song L: MACC1 as a prognostic biomarker for early-stage and AFP-normal hepatocellular carcinoma. PLoS One 8: e64235, 2013.

13. Wang Z, Li Z, Wu C, Wang Y, Xia Y, Chen L, Zhu Q and Chen Y: $\mathrm{MACC} 1$ overexpression predicts a poor prognosis for non-small cell lung cancer. Med Oncol 31: 790, 2014.

14. Zhu M, Xu Y, Mao X, Gao Y, Shao L and Yan F: Overexpression of metastasis-associated in colon cancer-1 associated with poor prognosis in patients with esophageal cancer. Pathol Oncol Res 19: 749-753, 2013.

15. Yang T, Kong B, Kuang YQ, Cheng L, Gu JW, Zhang JH, Shu HF, Yu SX, He WQ, Xing XM and Huang HD: Overexpression of MACC1 protein and its clinical implications in patients with glioma. Tumour Biol 35: 815-819, 2014.

16. Huang Y, Zhang H, Cai J, Fang L, Wu J, Ye C, Zhu X and Li M: Overexpression of MACC1 and Its significance in human breast cancer progression. Cell Biosci 3: 16, 2013.
17. Zhen T, Dai S, Li H, Yang Y, Kang L, Shi H, Zhang F, Yang D, Cai S, He Y, et al: MACC1 promotes carcinogenesis of colorectal cancer via $\beta$-catenin signaling pathway. Oncotarget 5: 3756-3769, 2014.

18. Quirke P, Williams GT, Ectors N, Ensari A, Piard F and Nagtegaal I: The future of the TNM staging system in colorectal cancer: Time for a debate? Lancet Oncol 8: 651-657, 2007.

19. Livak KJ and Schmittgen TD: Analysis of relative gene expression data using real-time quantitative PCR and the 2(-Delta Delta C(T)) Method. Methods 25: 402-408, 2001.

20. Xia Y, Zhu Y, Ma T, Pan C, Wang J, He Z, Li Z, Qia X and Chen Y: miR-204 functions as a tumor suppressor by regulating SIX1 in NSCLC. FEBS Letters 588: 3703-3712, 2014.

21. Kokoszyńska K, Kryński J, Rychlewski L and Wyrwicz LS: Unexpected domain composition of MACC1 links MET signaling and apoptosis. Acta Biochim Pol 56: 317-323, 2009.

22. Wang Z, Ma B, Ji X, Deng Y, Zhang T, Zhang X, Gao H, Sun H, Wu H, Chen X and Zhao R: MicroRNA-378-5p suppresses cell proliferation and induces apoptosis in colorectal cancer cells by targeting BRAF. Cancer Cell Int 15: 40, 2015.

23. Zhu M, Xu Y, Ge M, Gui Z and Yan F: Regulation of UHRF1 by microRNA-9 modulates colorectal cancer cell proliferation and apoptosis. Cancer Sci 106: 833-839, 2015

24. Boardman LA: Overexpression of MACC1 leads to downstream activation of $\mathrm{HGF} / \mathrm{MET}$ and potentiates metastasis and recurrence of colorectal cancer. Genome Med 1: 36, 2009.

25. Shirahata A, Shinmura K, Kitamura Y, Sakuraba K, Yokomizo K, Goto T, Mizukami H, Saito M, Ishibashi K, Kigawa G, et al: MACC1 as a marker for advanced colorectal carcinoma. Anticancer Res 30: 2689-2692, 2010. 